\title{
Hepatitis C Virus Reinfection after Sustained Virological Response in Those Who Continue to Inject Drugs at the Time of Treatment
}

\author{
Baxter $\mathrm{J}^{1,2^{*}}$, Verma $\mathrm{A}^{2}$ and Vilar $\mathrm{FJ}^{1}$
}

${ }^{1}$ Monsall Unit, Infectious Diseases Department, North Manchester Hospital, Delauneys Road, Crumpsall, Manchester, M8 5RB, UK

${ }^{2}$ Division of Population Health, Health Services Research and Primary Care, School of Health Sciences, Faculty of Biology, Medicine and Health, University of Manchester, Manchester Academic Health Science Centre, UK

*Corresponding author: Baxter J, Infectious Diseases Department, North Manchester Hospital, Delauneys Road, Crumpsall, Manchester, M8 5RB, United Kingdom, Tel: 0044 7930107761, Fax: 0044161 7202680, E-mail: jojoberry@hotmail.com

Citation: Baxter J, Verma A, Vilar FJ (2018) Hepatitis C Virus Reinfection after Sustained Virological Response in Those Who Continue To Inject Drugs at the Time of Treatment. J Gastroenterol Metabol 1: 103

Article history: Received: 11 November 2017, Accepted: 20 February 2018, Published: 21 February 2018

\begin{abstract}
Objective: Data regarding reinfection post sustained virological response (SVR) is starting to emerge but study numbers are limited. People who inject drugs (PWID) are responsible for the largest number of hepatitis C virus (HCV) transmissions in developed countries. Treatment in PWID is key to HCV elimination but concern about reinfection is a major barrier to offering treatment. This study aims to determine the reinfection rates in this high risk group.
\end{abstract}

Design: A total of sixty PWID post SVR with pegylated interferon and ribavirin were assessed annually. Those who injected in the 6 months prior to treatment were classified as the 'current' drug using group. Those who stopped injecting at least 6 months prior to treatment the 'past' drug using group.

Findings: The total duration of follow up post SVR in this study was 248.6 years. There were two cases of reinfection. The rate of reinfection in all participants was 0.80 cases per 100 person years ( $95 \%$ confidence interval [CI], 0.10 to 2.88 ). The total rate of reinfection in only the 'current' group was 1.34 cases per 100 person years (95\% CI, 0.16 to 4.76 ).

Conclusion: These low rates of reinfection, even in the highest risk injecting group, add further merit to prioritising provision of HCV treatment to PWID. Well tolerated directly acting antiviral (DAA) therapy with high SVR rates in PWID, will reduce the prevalence in this population as we move towards elimination of HCV.

Keywords: Hepatitis C Virus; Reinfection; Injecting Drug Use; Sustained Virological Response -SVR; People Who Inject Drugs - PWID

\section{Introduction}

Hepatitis $\mathrm{C}(\mathrm{HCV})$ is an RNA virus that predominantly affects the liver, although it can also affect the lymphatic, digestive, immune and nervous systems. HCV leads to inflammation, fibrosis and eventually cirrhosis of the liver if left untreated. It also increases the risk of hepatocellular carcinoma. People who inject drugs (PWID) account for more than 90\% of new HCV infections globally. The prevalence of anti-HCV antibodies varies between countries but the majority report prevalence estimates above 60\% [1]. HCV treatment in PWID has comparable sustained virological response (SVR) rates to those who do not inject drugs [2]. Modelling studies suggest that increasing the treatment rates among PWID could prevent onwards transmission and dramatically reduce HCV prevalence [3]. Treatment of PWID is recommended in international guidelines [4,5]. Despite these factors, there remain numerous barriers to provision of treatment in this population [6]. One such barrier is the perception of high rates of reinfection in PWID.

The main aim of this study was to determine if patients who have been successfully treated for HCV and who were currently injecting drug users at the time of treatment have comparable rates of reinfection to those who injected drugs in the past (but stopped at least 6 months before treatment start). 


\section{Key points}

- HCV reinfection rate in all 60 PWID was 0.80 cases per 100 person years (95\% CI, 0.10 to 2.88 )

- Reinfection rate in 'current' injecting group was 1.34 cases per 100 person years (95\% CI, 0.16 to 4.76$)$ and the reinfection rate in those who share injecting paraphernalia post treatment is 3.52 per 100 person years (95\% CI, 0.43 to 12.14 ).

-Demonstrates low reinfection rates even in the highest risk injecting groups.

- This study reinforces the need to prioritise future DAA treatment for PWID to have a discernible impact on incidence of HCV as we move towards HCV elimination.

\section{Methods}

A database at North Manchester Hospital details all patients started on Hepatitis C treatment since 2004. This database was accessed and those patients with injecting drug use at any time as their risk factor for acquisition of HCV were identified. Records of those who injected drugs in the 6 months prior to treatment were collated, including those who continued to inject during treatment. These were classed as the 'current' drug using group. Another group of patients that stopped injecting drugs at least 6 months prior to commencing treatment were identified as the 'past' drug using group. Telephone contact was attempted in the first instance on at least two separate occasions. If successful the study was verbally explained and information was sent via mail for them to read through. A second telephone contact was conducted to determine if they wished to participate and to organise a consent visit if they did. There were twelve (20\%) participants recruited via this method. If the initial telephone contact was unsuccessful any new contact details were ascertained from their General Practitioner. Written information and a study invitation letter were sent via mail to them. The remaining 48 (80\%) participants were recruited via this method.

A total of 45 current IDUs were invited to participate. We identified a total of 46 from the whole database but one had died. A matched number of past IDUs were invited to participate. Participants were compensated for their time with a payment of $£ 30$ cash per visit to cover travel expenses.

The first visit involved a full discussion of the study and then written informed consent was obtained. Out of the 60 patients who attended for their first visit, 32 (53\%) of them attended at least one year later for a second visit. Of these 32 patients, 19 (59\%) were from the current IDU group. The following inclusion criteria were used in this study: aged 18 years or older, previous HCV treatment achieving SVR, a history of intravenous drug use and the participant must be able to give informed consent. The following exclusion criteria were used: Co-infection with hepatitis B or HIV, participants must not be in prison. Pregnant females were excluded as well any participant who did not achieve SVR with treatment. If they were unable to give informed consent they were also excluded.

\section{Data Collected}

Patients were followed up to collect data on outcomes including reinfection rates using annual HCV RNA (with subsequent genotyping if HCV RNA positive) and liver disease progression using standard liver function blood tests and a Fibroscan ${ }^{\mathrm{Tm}}$ (a noninvasive method to assess liver fibrosis and cirrhosis). The participants were asked to attend for 2 visits in total at least one year apart. Any positive HCV RNA results were confirmed by subsequent testing and referred for further genotype testing and were offered treatment.

A questionnaire regarding drug use post treatment was completed to detail any high risk practises. They were also questioned again about drug use during treatment in order to confirm which group of drug use they should be assigned to and to reduce the possibility of previous underreporting due to fear of exclusion from the initial treatment programme. After completion of the questionnaire, safe injection technique was discussed with each participant. Alcohol intake was also assessed using the cut off of 21 units per week for males and 14 units per week for females [7].

\section{Statistical analysis}

Demographic data is summarised using descriptive statistics. Incidence rates for reinfection are presented as cases per 100 person years and binomial 95\% confidence intervals are given. The reinfection rates have been calculated for all PWID and separately for the 'current' group to allow comparison. The SPSS statistics Version 22 (IBM, Chicago, Illinois, USA) was used to summarise the health domains data and logistic regression analyses were performed to examine factors associated with fibrosis. Two-sided $\mathrm{p}$ values $<0.05$ were considered statistically significant.

\section{Ethics}

This study was reviewed by and given a favourable ethical opinion by the National Research Ethics Service North West - Greater Manchester South Ethics Committee in 2011 (NW/11/0258). All patients provided written consent prior to any study related participation. 


\section{Results}

A total of 46 current drug users were identified from the database, of which one had died. 45 current drug users were therefore invited to take part in the study. 36 were screened and consented to take part. This equates to an $80 \%$ uptake which was better than anticipated. A matched number (45) of past injectors were invited. 24 were subsequently screened and consented to take part. The uptake in this group was less, $53 \%$.

In those patients who injected up to and at the time of treatment (the 'current' group), 38.9\% (14 out of 36) stopped injecting drugs after treatment and did not restart. $61.1 \%$ continued to inject drugs post treatment, of which $59 \%$ admitted to sharing of injecting paraphernalia. None of the 'past' drug using group restarted injecting during or after treatment. Examining the 'past' group and their injecting habits it can be seen that $62.5 \%$ of this group admitted to sharing injecting paraphernalia when they did inject. The most commonly injected drug was heroin in $83.3 \%$, followed by crack cocaine in $40 \%$ and amphetamines in $6.7 \%$. The median age was 43 years (Table 1 ).

\begin{tabular}{|c|c|c|c|c|c|}
\hline & & CURRENT $(n=36)$ & CURRENT (\%) & PAST $(n=24)$ & PAST (\%) \\
\hline \multirow[t]{5}{*}{ AGE } & $<30$ & 0 & 0 & 2 & 8.3 \\
\hline & $31-40$ & 14 & 38.9 & 6 & 25.0 \\
\hline & $41-50$ & 15 & 44.4 & 11 & 45.8 \\
\hline & $51-60$ & 4 & 11.1 & 4 & 16.7 \\
\hline & $>60$ & 2 & 5.6 & 1 & 4.2 \\
\hline \multirow[t]{2}{*}{ SEX } & male & 33 & 91.7 & 17 & 70.8 \\
\hline & female & 3 & 8.3 & 7 & 29.2 \\
\hline \multirow[t]{5}{*}{ BMI } & $<20$ & 1 & 2.8 & 1 & 4.2 \\
\hline & 20 to 24.9 & 15 & 41.7 & 12 & 50.0 \\
\hline & 25 to 29.9 & 13 & 36.1 & 5 & 20.8 \\
\hline & 30 to 34.9 & 6 & 16.7 & 4 & 16.7 \\
\hline & $>35$ & 1 & 2.8 & 2 & 8.3 \\
\hline \multirow[t]{4}{*}{ GENOTYPE } & 1 & 12 & 33.3 & 2 & 8.3 \\
\hline & 2 & 1 & 2.8 & 0 & 0.0 \\
\hline & 3 & 23 & 63.9 & 22 & 91.7 \\
\hline & 4,5 or 6 & & 0.0 & 0 & 0.0 \\
\hline \multirow[t]{2}{*}{ ALCOHOL } & Above limits for sex & 4 & 5.9 & 6 & 25.0 \\
\hline & Below limits for sex & 32 & 94.1 & 18 & 75.0 \\
\hline
\end{tabular}

A Fibroscan was performed at each visit. A cut off of 12.5kPa as the F3/F4 staging was used to define significant fibrosis/cirrhosis (as suggested by Echosense, the manufactures of the Fibroscan ${ }^{\mathrm{Tw}}$ ) and $14.5 \mathrm{kPa}$ to represent more definitive F4 staging. A total of $10 \%$ of this cohort had a Fibroscan score above $12.5 \mathrm{kPa}$ representing significant fibrosis/cirrhosis. A further $20 \%$ of the participants had

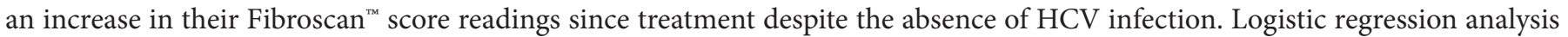
was performed to examine for any potential statistically significant factors affecting fibrosis. The difference in Fibroscan ${ }^{\mathrm{T}}$ readings, $^{\mathrm{s}}$ age, sex, alcohol consumption (above or below recommended limits), genotype and injecting status (current or past group) were all entered into the analysis. There were no factors analysed that were associated with a statistically significant effect on fibrosis progression in this cohort of patients post SVR.

There were two cases of reinfection identified in this study, both in the 'current' injecting group (Table 2). The total duration of participant follow up post SVR in all 60 participants was 248.6 years (mean follow up of 50 months, range 11 months to 95 months). The total rate of reinfection in all participants was 0.80 cases per 100 person years $(2 / 248.6=0.008 \times 100=0.80)$. The $95 \%$ confidence intervals are 0.001 to 0.0288 ( 0.10 cases per 100 person years to 2.88 cases per 100 person years).

The total duration of patient follow up in only the 'current' group (36 participants) was 149 years. The total rate of reinfection in only the 'current' group was 1.34 cases per 100 person years $(2 / 149=0.0134 \times 100=1.34)$. The $95 \%$ confidence intervals are 0.0016 to 0.0476 ( 0.16 cases per 100 person years to 4.76 cases per 100 person years).

The total duration of patient follow up in only those in the current group with ongoing intravenous drug use post treatment (19 participants, 59\% of the current group) was 90 years. The rate of reinfection in these participants (61\% of the current group) was 2.22 cases per 100 person years $(2 / 90=0.222 \times 100=2.22)$. The $95 \%$ confidence intervals are 0.27 to 7.2 cases per 100 person years. If we then further examine those who continue to inject post treatment and continued to share injecting paraphernalia, making their injecting practises very high risk, the total duration of patient follow up is 56.83 years. The rate of reinfection is 3.52 per 100 person years $(2 / 56.83=0.0352 \times 100=3.52)$. The $95 \%$ confidence intervals are 0.43 to 12.14 cases per 100 person years.

Demographic details of the 'current' and 'past' groups are shown in Table 1. 


\section{Reinfection Cases}

The two cases of reinfection were both reinfected with a different genotype to the original one cleared with treatment. Case one, participant number 10, was originally infected with genotype 1 hepatitis $\mathrm{C}$ and treated and achieved SVR four years prior to entry into this study. At study visit 1 he remained HCV negative. However, at visit 2 his HCV RNA was 3725 IU/ml. The reinfection genotype was confirmed as genotype 3. Multiple attempts to contact him through telephone and letter failed. His GP was also contacted. All attempts to reassess the patient have been unsuccessful to date. It is therefore unknown if this is a persistent reinfection with sustained viraemia requiring further treatment; or if in fact he has managed to spontaneously clear the genotype 3 HCV reinfection.

\begin{tabular}{|c|c|c|}
\hline & Participant 10 & Participant 48 \\
\hline Age (visit 1) & 35 & 55 \\
\hline Sex & Male & Male \\
\hline Genotype prior to 1st SVR & 1 & 3 \\
\hline Current or past group & Current & current \\
\hline $\begin{array}{c}\text { Injecting frequency pre/during } \\
\text { 1st treatment }\end{array}$ & Daily & weekly \\
\hline $\begin{array}{l}\text { Injecting frequency post 1st } \\
\text { SVR }\end{array}$ & Monthly & less than monthly \\
\hline Visit 1 HCV RNA (IU/ml) & $<12$ & 3725 \\
\hline Visit 2 HCV RNA (IU/ml) & 2569854 & N/A \\
\hline Reinfection genotype & 3 & 1 \\
\hline Pretreatment Fibroscan ${ }^{\mathrm{rat}}$ & N/A & 8.6 \\
\hline Visit 1 Fibroscan $^{\text {mis }}$ & 6.9 & 7.5 \\
\hline Visit 2 Fibroscan $^{\mathrm{Tn}}$ & 8 & N/A \\
\hline Alcohol units per week & 3 & 112 \\
\hline Type of alcohol & Lager & strong cider/wine \\
\hline $\begin{array}{l}\text { Maximum Time between SVR } \\
\text { and reinfection (months) }\end{array}$ & 48 & 30 \\
\hline Drugs injected & heroin and cocaine & heroin \\
\hline Sharing paraphernalia & every week & every week \\
\hline Outcome & $\begin{array}{l}\text { Invited to attend for } \\
\text { assessment and retreatment } \\
\text { consideration but did not } \\
\text { attend }\end{array}$ & $\begin{array}{l}\text { Retreated with } \\
\text { bocepravir, ribavirin } \\
\text { and pegylated } \\
\text { interferon. }\end{array}$ \\
\hline Reinfection outcome & $\begin{array}{l}\text { Uncertain Spontaneous } \\
\text { clearance Persistent } \\
\text { reinfection }\end{array}$ & SVR achieved \\
\hline
\end{tabular}

The second case of reinfection, participant number 48, was initially infected with genotype 3 Hepatitis $\mathrm{C}$ and treated and achieved SVR thirty months prior to study entry. It is noteworthy that he consumed 112 units of alcohol per week at study entry. He also admitted to sharing injecting paraphernalia on a weekly basis. At visit 1 his HCV RNA was 2569854 IU/ml. On this occasion he was reinfected with HCV genotype 1. This participant was reassessed and a repeat HCV RNA at least 6 months later confirmed persistent reinfection. He was referred back to the hepatitis team at the hospital. He agreed to be retreated for hepatitis $\mathrm{C}$ with pegylated interferon, ribavirin and bocepravir. His 12 week and 24 week post treatment HCV RNA were both negative, confirming a sustained virological response, as seen in Table 2.

\section{Discussion}

The rate of reinfection in this study was low, in line with similar studies detailed below. This is the first study that has looked at reinfection in those who actively inject drugs in the 6 months leading up to and during treatment, without the need for opiate substitution therapy (OST) initiation as a criterion for treatment, and to compare this to a 'past' drug using group in the same centre. In other reinfection studies there has been a need for abstinence from injecting for 6 months prior to treatment or the use of OST. In this study all patients in the 'current' group were included in the reinfection analysis.

A key strength of this study is the high inclusion rate (80\%) in the current drug using group treated at our centre. This has minimised any potential selection bias. This study also provides one of the longest follow-up periods in any reinfection study to date. The inclusion of the past drug using group strengthened the study design. One possible explanation for the lower uptake in the past drug using group to this study could be that HCV is no longer a priority in their life, especially if they stopped injecting 
many years ago and perceive their risk of reinfection to be very low or zero. In the study population, 59\% of those who continued to inject post treatment continued to share injecting paraphernalia. Despite this they did not become reinfected with Hepatitis C.

In our study, in the group who had been abstinent from injecting for 6 months prior to treatment there were no cases of reinfection. Furthermore, no one resumed injection drug use in this group. This cut off of 6 months abstinence was an excellent predictor of reduced risk of relapse to injecting drug use in this population. Over half of those who did inject post treatment shared paraphernalia. It is vital that harm reduction measures are included in the treatment package for 'current' PWID. This will support future provision of DAA therapy in this group and help address reinfection concerns.

This study additionally supports a low rate of reinfection in the 'current' drug injecting population. This remains true even when selecting out those who have high risk injecting practises. Those who continue to inject post treatment and continue to share paraphernalia (19 participants) have a reinfection rate of 3.52 per 100 person years ( $95 \%$ confidence intervals 0.43 to 12.14 cases per 100 person years). Targeting this population for treatment is needed, rather than putting up barriers to treating them.

Lewis, et al., presented reinfection data in 2011 [8]. Their cohort was based in nurse led community addiction services in London and $59 \%$ actively injected drug at the time of treatment. Only one case of reinfection was seen in the 81 patients who received interferon based therapy. It is not stated if this case was an actively injecting drug user at the time of start of treatment or not. Length of follow up is also not detailed. Nonetheless, it adds further evidence that the rate of reinfection is low in this population.

Grebely, et al., reported a trial of directly observed interferon based therapy in patients with a history of injecting drug use, in which $46 \%$ relapsed to injecting drugs post treatment [9]. There were two cases of reinfection seen, with one of those spontaneously clearing the infection. This equates to 5.3 cases per 100 person years (95\% CI, 0.6-19.2).

Dalgard, et al., also looked at reinfection rates post SVR with interferon based treatment [10]. This Norwegian population all had 6 months abstinence from injecting drug use prior to treatment. The rate of persistent reinfection in the 37 patients who relapsed to injecting drug post treatment was 4.9 cases per 100 person years (95\% CI, $2.3-8.9)$. The rate of relapse to injecting drug use is considerably higher than in our study where no patient who had been abstinent from injecting drug use for 6 months prior to treatment relapsed to injecting drug use.

A meta-analysis by Aspinal, et al., reported similarly low rates of reinfection [11]. In those patients who continued injecting drug use post treatment, the reinfection rate was 6.4 cases per 100 person years (95\% CI, 2.5-16.7).

Weir, et al., used opiate or injection related hospital admission post SVR as a proxy for ongoing active drug use in their study [12]. This sub section of their cohort had a reinfection rate of 5.68 per 100 person years (95\% CI, 1.84-13.26).

Marco, et al., looked at reinfection in those who were treated and obtained SVR whilst in prison [13]. 10.1\% of their cohort injected drugs during or after treatment. In this group the rate of reinfection was 33.01 cases per 100 person years (95\% CI, 11.4 to 60.1).

Further data to support these low reinfection rates is starting to emerge from DAA studies that include PWID. An example is Merck's C-Edge Co-Star trial that looked at the safety and efficacy of Elbasvir/Grazoprevir in patients who inject drugs [2]. All participants had to be receiving opiate substitution therapy. Active injecting drug use was not an exclusion criterion and urine drug screens were collected throughout treatment to confirm ongoing use. SVR 12 rates of $95.5 \%$ to $96.6 \%$ were achieved. There were 6 cases of probable reinfection in this study. The total duration of patient years follow in this study was 130.6 years. This equates to 4.6 reinfections per 100 person-years (95\% CI, 1.7 to 10.0$)$.

SVR rates in excess of $95 \%$ have now been seen across all genotypes with the DAAs including the harder to treat groups such as cirrhotic patients and previous null responders to treatment. The biggest barrier to providing all patients with the new DAAs is cost.

A key limitation of this study is the frequency of sampling for HCV RNA. Annual sampling may miss those reinfections that the patient clears spontaneously and the incidence of all reinfection episodes is likely to be higher. However, it is those with persistent viraemia and therefore persistent reinfection that are important to quantify. This is because much of the physicians' reluctance to treat 'current' drug users comes from the perception that reinfection will occur resulting in the need for further repeated courses of treatment. Whilst those transient reinfections that we may miss are very important in terms of onwards transmission, they do not result in the need for further treatment.

Another limitation is that data collected retrospectively from patient notes could be subject to information bias due to under reporting of injecting behaviour at the time of first treatment. Furthermore the self-reported behaviour data gathered in the drug and alcohol questionnaires could be subject to recall bias. A further limitation was the decision not to use urine drug screens. The use of urine drug screens at visits 1 and 2 would have helped confirm the use of any illicit drugs, rather than relying on selfreported behaviour. The fact that each participant received interferon based therapy, which can be associated with severe side effects may have also impacted on risk behaviour. It is possible that a larger number of current PWID may have stopped injecting post treatment if treated with directly acting antivirals (DAAs) that are not associated with such severe side effects. A limitation in the Fibroscan $^{\mathrm{Tm}}$ data is the time period between visits. The maximum interval between each Fibroscan ${ }^{\mathrm{Tm}}$ was 32 months. A single operator was used to perform all the Fibroscans ${ }^{\text {TM }}$ to reduce inter operator variability. 
Larger and longer follow up trials post SVR after DAAs in PWID are urgently needed. The cases of new infections in an area where PWID are treated with DAAs need to be examined to determine the emergence of resistance associated variants in that population and the potential clinical relevance of these. This additional evidence regarding reinfection in PWID post DAA treatment will support the scale up of treatment needed to have a discernible impact on HCV prevalence, as we move towards the goal of HCV elimination.

\section{Acknowledgement}

Financial disclosure: Unrestricted grant of $£ 12,200$ received from Roche Pharmaceuticals to conduct the research.

Clinical Trial Number: NCT01329952

\section{References}

1. Nelson PK, Mathers BM, Cowie B, Hagan H, Jarlais DD, et al. (2011) Global epidemiology of hepatitis B and hepatitis C in people who inject drugs: results of systematic reviews. The Lancet 378: 571-83.

2. Dore GJ, Altice F, Litwin AH, Grebely J, Dalgard O, et al. (2016) C-Edge Co-Star: Risk of reinfection following successful therapy with Elbasvir and Grazoprevir in persons who inject drugs (PWID) receiving opioid agonist therapy (OAT). J Hepatol 64: S771.

3. Martin NK, Vickerman P, Grebely J, Hellard M, Hutchinson SJ, et al. (2013) Hepatitis C virus treatment as prevention among people who inject drugs: Modelling treatment scale up in the age of directly acting anti-virals. Hepatology 58:1598-609.

4. European Association for the Study of the Liver (2015) EASL recommendations on the treatment of hepatitis C 2015. J Hepatol 63: $199-236$.

5. AASLD/IDSA HCV Guidance Panel (2015) AASLD-IDSA recommendations for testing, managing and treating adults infected with Hepatitis C virus 2015. Hepatology 62: 932-54.

6. Inversen J, Grebely J, Topp L, Wand H, Dore G, et al. (2014) Uptake of hepatitis C treatment among people who inject drugs attending Needle and Syringe Programs in Australia, 1999-2011. J Viral Hepatitis 21: 198-207.

7. Alcohol guidelines (2012) House of commons, science and technology committee. Eleventh report of session 2010-2012.

8. Lewis H (2011) Actively injecting drug users can be successfully treated with antiviral therapy for HCV, are unlikely to be re-infected, and significantly reduce their illicit drug use. European Association for the Study of the Liver Conference 2011.

9. Grebely J, Knight E, Ngai T, Genoway KA, Raffa JD, et al. (2010) Reinfection with hepatitis C virus following sustained virological response in injection drug users. J Gastroenterol Hepatol 25: 1281-4.

10. Dalgard O, Midgard H, Bjoro B, Mæland A, Konopski Z, et al. (2016) Hepatitis C reinfection after sustained virological response. J Hepatol 64: 1020-6.

11. Aspinall EJ, Corson S, Doyle JS, Grebely J, Hutchinson SJ, et al. (2013) Treatment of hepatitis C virus amongst people who are actively injecting drugs: a systematic review and meta-analysis. Clin Infect Dis 57: S80-9.

12. Weir A, McLeod A, Innes H, Valerio H, Aspinall EJ, et al. (2016) Hepatitis C reinfection following treatment induced viral clearance among people who have injected drugs. Drug Alcohol Depend 165: 53-60.

13. Marco A, Esteban JI, Sole C, da Silva A, Ortiz J, et al. (2013) Hepatitis C virus infection among prisoners with sustained virological response after treatment for chronic hepatitis C. J Hepatol 59: 45-51. 